\title{
Effects of Acrophobic Fear and Trait Anxiety on Human Behavior in a Virtual Elevated Plus-Maze
}

\section{OPEN ACCESS}

Edited by:

Barbara Rothbaum,

Emory University, United States

Reviewed by:

Mark Steven Burton,

Emory University, United States

Brian Dixon,

New Zealand Psychological Society,

New Zealand

Katarzyna Wyka,

City University of New York,

United States

${ }^{*}$ Correspondence:

Octavia Madeira

octavia.madeira@uni-wuerzburg.de

Specialty section:

This article was submitted to Virtual Reality in Medicine,

a section of the journal

Frontiers in Virtual Reality

Received: 29 November 2020

Accepted: 18 February 2021

Published: 20 April 2021

Citation:

Madeira O, Gromer D, Latoschik ME and Pauli $P$ (2021) Effects of Acrophobic Fear and Trait Anxiety on Human Behavior in a Virtual

Elevated Plus-Maze.

Front. Virtual Real. 2:635048.

doi: $10.3389 /$ frvir.2021.635048

\author{
Octavia Madeira ${ }^{1 *}$, Daniel Gromer ${ }^{1}$, Marc Erich Latoschik ${ }^{2}$ and Paul Pauli ${ }^{1,3}$ \\ ${ }^{1}$ Department of Psychology I, Biological Psychology, Clinical Psychology and Psychotherapy, University of Würzburg, Würzburg, \\ Germany, ${ }^{2}$ Department of Human-Computer-Interaction, University of Würzburg, Würzburg, Germany, ${ }^{3}$ Center of Mental Health, \\ University of Würzburg, Würzburg, Germany
}

The Elevated Plus-Maze (EPM) is a well-established apparatus to measure anxiety in rodents, i.e., animals exhibiting an increased relative time spent in the closed vs. the open arms are considered anxious. To examine whether such anxiety-modulated behaviors are conserved in humans, we re-translated this paradigm to a human setting using virtual reality in a Cave Automatic Virtual Environment (CAVE) system. In two studies, we examined whether the EPM exploration behavior of humans is modulated by their trait anxiety and also assessed the individuals' levels of acrophobia (fear of height), claustrophobia (fear of confined spaces), sensation seeking, and the reported anxiety when on the maze. First, we constructed an exact virtual copy of the animal EPM adjusted to human proportions. In analogy to animal EPM studies, participants $(N=30)$ freely explored the EPM for $5 \mathrm{~min}$. In the second study $(N=61)$, we redesigned the EPM to make it more human-adapted and to differentiate influences of trait anxiety and acrophobia by introducing various floor textures and lower walls of closed arms to the height of standard handrails. In the first experiment, hierarchical regression analyses of exploration behavior revealed the expected association between open arm avoidance and Trait Anxiety, an even stronger association with acrophobic fear. In the second study, results revealed that acrophobia was associated with avoidance of open arms with mesh-floor texture, whereas for trait anxiety, claustrophobia, and sensation seeking, no effect was detected. Also, subjects' fear rating was moderated by all psychometrics but trait anxiety. In sum, both studies consistently indicate that humans show no general open arm avoidance analogous to rodents and that human EPM behavior is modulated strongest by acrophobic fear, whereas trait anxiety plays a subordinate role. Thus, we conclude that the criteria for crossspecies validity are met insufficiently in this case. Despite the exploratory nature, our studies provide in-depth insights into human exploration behavior on the virtual EPM.

Keywords: elevated plus-maze, EPM, anxiety, virtual reality, translational neuroscience, acrophobia, trait anxiety

\section{INTRODUCTION}

The Elevated Plus-Maze (EPM) is a widely used and well-accepted apparatus to measure anxiety in rodents. Its advantage is that it assesses natural behavior and therefore does not require a previous conditioning process or other preparations that might interfere with experimental variables distorting behavioral outcomes. The EPM, with its open and closed arms, comprises a conflict 
situation as rodents, on the one hand, prefer dark and enclosed arms to avoid potential predators, but, on the other hand, are naturally prone to engage actively in exploration behavior towards novel stimuli (Barnett, 2017). Following-up a study of Montgomery (1955), Pellow et al. (1985) developed an elevated plus-shaped platform with two closed and two open arms. They examined rats and observed that anxiolytic drugs (e.g., diazepam) significantly increased open arm entries and open arm exploration time, whereas anxiogenic medication induced opposite effects.

These seminal findings strongly suggest that rodents' movement patterns in the EPM are reliable and valid indicators of general anxiety. Based on these findings and numerous follow-up studies, the EPM is now considered a standard test of anxiety in rodents and routinely used in preclinical translational research, i.e., for drug or target gene testing. For instance, the anxiolytic effect of benzodiazepines was detected in rodents by using the EPM, and this is why diazepam is now an established anxiolytic drug within the human clinical setting until today.

One way to examine the translational validity of animal tests is re-translation, i.e., the development of human analogs to animal paradigms (Kirlic et al., 2017). Such re-translation proved to be successful for several cognitive tasks, e.g., monetary- or punishment-based conflict paradigms. First attempts to retranslate animal paradigms assessing anxiety-related behavior proved to be successful too (Kirlic et al., 2017). An advantage of such re-translational human tasks is that they assess both behaviors analogous to animal paradigms and verbal responses which may help to validate the paradigm. For instance, Aupperle et al. (2011) re-translated an approach-avoidance conflict task frequently used in anxiety researches in animals to humans and found that either conflict or reward approach is associated with certain aspects of anxiety sensitivity including gender. Walz et al. (2016) re-translated the open field test, which is frequently used in rodents to measure anxiety. In rodents, increased thigmotaxis during exploration of the open field, i.e., staying close to the open field's wall, is interpreted as an indicator of animal anxiety (Gould et al., 2009). In analogy, Walz et al. asked their participants to freely explore an open field, i.e., a soccer field surrounded by trees and bushes (Walz et al., 2016). Results revealed that individuals reporting agoraphobic fear or high levels of anxiety sensitivity, a known risk factor for agoraphobia and panic disorder (Hofmann et al., 2009), displayed an increased thigmotaxis, i.e., they moved along the walls of the open field more and avoided its center for a longer time. These results validate the open field animal studies because they corroborate an association between reported level of anxiety and open field behavior (Grillon and Ernst, 2016). Interestingly, these findings also imply that characteristic ethologic patterns in rodents are conserved in humans also and seem to play a significant role in human anxiety.

However, there still exists a scientific gap regarding the crossspecies translational validity of these anxiety models. It is often criticized that they lack predicational clinical value (Grillon and Ernst, 2016). As a result, there is a paucity in developing new anxiolytic agents or even therapeutic approaches based on these preclinical "classic" tests (Griebel and Holmes, 2013; Grillon et al., 2019). At the same time, the (re-) translation of Pavlovian fear conditioning already provided numerous insights to fear-related issues in humans while maintaining methodological comparability and providing cross-species validity for years by now (Lonsdorf et al., 2017; Haaker et al., 2019). Consequently, closing this gap by testing cross-species validity could similarly set new impulses in both human and animal research.

Trait anxiety is an enduring and generalized predisposition to react with fear in potentially threatening or ambiguous situations (Spielberger, 1966; Spielberger, 2013). It was found that individuals with high trait anxiety scores are prone to develop an anxiety disorder over their lifetime (Hofmann et al., 2009; Gallagher et al., 2014; Kindt and Soeter, 2014). On a behavioral level, high trait anxiety manifests in harm avoidance, i.e., avoidance behavior (Maner and Schmidt, 2006). From a clinical point of view, findings on emotional and behavioral mechanisms of subthreshold anxiety disorders (i.e., not yet pathologic but close to meet diagnostic criteria) imply that it can be similarly impairing as a fully developed pathologic condition (Carter et al., 2001; Karsten et al., 2011). In light of this scientific paucity, the human virtual EPM might be a promising tool to investigate behavioral movement patterns associated with trait anxiety and contribute to a translational understanding of anxiety mechanisms. Moreover, the insights on movement behavior related to trait anxiety might complement current research methods such as self-report data and physiological measures.

Virtual reality (VR) offers promising advancement in the development of human analogs to animal paradigms. VR is a computer-generated environment simulating sensory input, mostly visual and auditory, in which the participant is immersed, feels present, and can behave and interact with the environment (see Sanchez-Vives and Slater, 2005, for a discussion of its use in animal and human research; Bohil et al., 2011). For instance, Dobricki and Pauli (2016) used virtual reality to assess body-environment interactions and emotions in a virtual height scenario in humans, and Gromer et al. (2019) systematically examined the emotional responses of humans confronted with virtual heights.

Biedermann et al. (2017) adopted a mixed virtual reality design to develop a human elevated plus-maze (EPM) paradigm (see also Madeira et al., 2017). Their virtual EPM consisted of a real-world wooden maze providing haptic feedback combined with a virtual maze providing visual feedback presented with a head-mounted display (HMD). The maze was placed on a virtual rocky mountain surrounded by water with two opposite arms, and the center of the maze was surrounded by rocks while the other two arms reached out over the water at $55 \mathrm{~m}$ height. Results revealed that participants avoided open arms and reported more anxiety on these arms. Significantly, open arm avoidance was positively associated with acrophobic fear but not with anxious temperament (STAI) or social anxiety and negatively associated with sensation seeking. These opposing associations of acrophobia and sensation seeking with open arm avoidance behavior correspond with assumptions that both constructs are related to opposing motivational systems (Boecker and 
Pauli, 2019). Finally, anxioselective drugs were found to decrease and increase open arm avoidance as expected. While this study has several strengths, it also has important limitations. First, walls surround the closed arms of the rodent EPM, while the closed arms of the designed human EPM had no walls. Instead, the humans stood in both conditions on real wooden planks with tangible borders that in one condition were perceived visually as lying on rocky ground and in the other condition as high above the water. Second, the used HMD caused a dissociation of haptic from visual feedback as it precluded the perception of the own body including the feet. In consequence, participants' movement on the maze might have been unnatural requiring increased attention on the feet and floor, and this might have exaggerated the height perception and in consequence feelings of insecurity and arousal especially on the "open" arms. Finally, the rodent EPM behavior was found to be independent of height (Treit et al., 1993) and is therefore considered as a measure of general anxiety, e.g., it correlates with other anxiety measures like the open field test or the dark-light box (Ramos et al., 2008). In contrast, the observed human EPM behavior was related to fear of height, but not trait anxiety. In sum, the author's conclusion that the "identical outcome parameters in the human and rodent EPM facilitate translational research across species" (Biedermann et al., 2017 p8) might be premature.

Here, we examined the construct validity of a virtual EPM to assess the anxiety in humans in two studies. However, in contrast to Biedermann et al. (2017), we used a 5-sided Cave Automatic Virtual Environment (CAVE) instead of an HMD to present the virtual environment with the advantage that it allows participants to see the own body while simultaneously being able to walk naturally. In our opinion, this better allows free exploration comparable to a rodent EPM. Moreover, a CAVE system allows a bigger field of view and triggers less simulator sickness than an HMD (Rebenitsch and Owen, 2016), which might be a moderating variable for anxiety if using VR scenarios with height situations. Also, we decided to stick to the animal EPM design as close as possible and maintain the experimental procedure. Thus, the typical animal EPM (Komada et al., 2008) was enlarged to human proportions, and participants were asked to freely explore this environment while their behavior was recorded. In addition, we used ratings to assess anxiety at different EPM locations, and questionnaires were used to evaluate trait anxiety, acrophobic fear, and sensation seeking. Based on the findings in the first study and on participants' feedback, we decided to modify the virtual EPM in a second study and re-tested our hypotheses. Assuming that a human EPM allows behavioral assessment of anxiety in humans in analogy to rodent studies, we hypothesized that 1) humans exhibit a general open arm avoidance and that 2) their level of trait anxiety is positively associated with open arm avoidance and fear ratings on open arms. Alternatively, we speculated following Biedermann et al. (2017) that 3) acrophobic fear is positively associated with open arm avoidances and fear ratings on open arms and that 4) sensation seeking is negatively associated with open arm avoidance.

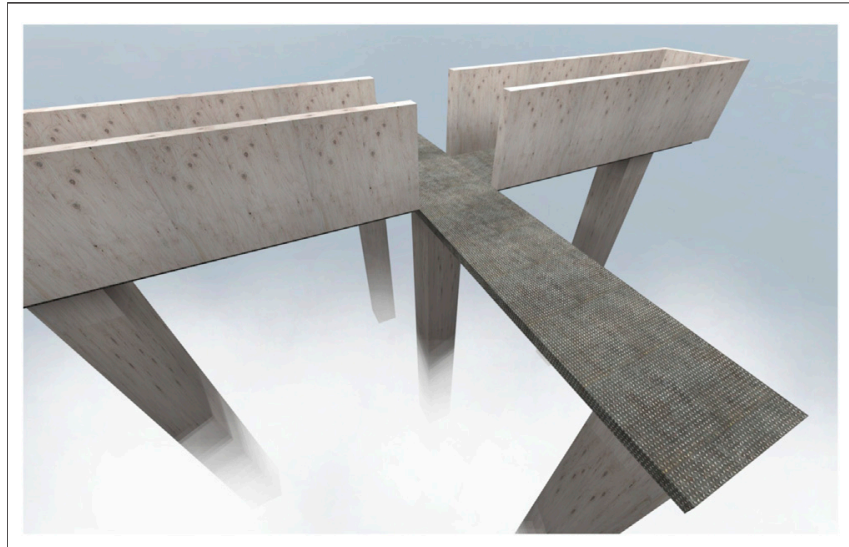

FIGURE 1 | Screenshot of the virtual Elevated Plus-Maze. Center area marks the starting point of the exploration phase lasting for $5 \mathrm{~min}$; starting gaze direction was randomized across participants.

\section{STUDY 1}

\section{Methods \\ Sample}

A total of 33 individuals, most of them students, were recruited via email and flyers and received either 12 EUR or course credit for participation. Due to technical problems during data recording, three participants were excluded from analysis resulting in a final sample of 30 individuals (10 males and 20 females) with a mean age of $M=21.93(S D=2.85)$.

The study was carried out following the recommendations of the Declaration of Helsinki and the Ethical Guidelines of the German Psychological Society. The Ethics Committee of the Department of Human-Computer-Interaction of the University of Würzburg provided ethical approval for the protocol.

\section{Apparatus}

Assessments were conducted in the 5-sided 3D Multisensoric PsyCave Laboratory of the Department of Psychology I of the University of Würzburg sized $4 \times 3 \times 2.95 \mathrm{~m}$. The visual simulations on the five canvases (4 walls plus floor) were presented via six projectors (NW-7, BARCO, Kuurne, Belgium) with a resolution of $1920 \times 1,200$ pixels each and connected to two computers per projector (Intel Core i7-2600K; 8GB RAM; Nvidia Geforce GTX 580; OCZ Vertex2 SSDs). Participants wore passively color-filtering glasses (Infitec Premium, Infitec, Ulm, Germany) for stereoscopic effect. Auditory instructions were given via a 7.1 surround sound system (CANTON, Weilrod, Germany). Motion tracking was recorded with four LED infrared cameras installed on top of the CAVE canvases (PhaseSpace Impulse, PhaseSpace Inc, San Leandro, CA, United States). A wireless Xbox 360 controller (Microsoft, Redmond, WA, United States) was used for navigation over long distances. Walking by foot was possible within the space of the CAVE.

The experimental virtual environment was set up with a selfmade modification of the first-person shooter game, "Half Life 2: Deathmatch" (Valve, Bellevue, Washington, United States) based 
on Source Engine 2007. The experimental procedure was managed by the CS-Research 5.6 software (VTplus, Würzburg, Germany; see www.cybersession.info for detailed information) (cf. Kinateder et al., 2014).

\section{Virtual Scenario}

The virtual human EPM was identical to the animal EPM but adjusted to human proportions. Thus, the plus-shaped platform had a height of $10 \mathrm{~m}$ with four maze arms arranged orthogonally to each other, each $11 \mathrm{~m}$ long and $3 \mathrm{~m}$ wide. To control for environmental cues that might interfere with exploration behavior and to exacerbate height estimation for participants, platform pillars were surrounded by fog. All arms had a solid ground texture. The two open arms were free of any visible barrier, whereas the two closed arms had wooden-textured walls of $3 \mathrm{~m}$ in height (see Figure 1). Please note that we were unaware of Biedermann et al.'s (2017) design as we conducted the study prior to their publication (Madeira et al., 2017).

\section{Tracking Data}

The participants' positioning data within the virtual scenario were tracked continuously with a sample rate of $60 \mathrm{~Hz}$. Based on this positioning data, we calculated the behavioral parameters during the $5 \mathrm{~min}$ of exploration time: time spent on open and closed arms, distance walked on open and closed arms, and total walking distance. The center area was defined as a transition zone and therefore excluded from analyses (Hogg, 1996).

\section{Anxiety and Presence Ratings}

After the free exploration and fading out of the virtual environment, participants were teleported to the center area of the maze again and asked to walk forward to a green footprint on one of the arms and then rate the anxiety experienced on that position with Subjective Units of Discomfort Scales (SUDS) ranged from 0 to 100 . Footprint markers were used to ensure consistency in position for ratings across participants. These ratings were assessed on one open and one closed arm selected randomly. After the anxiety rating trial, the feeling of presence was also assessed with the question "To which extent do you feel present in the VE, i.e., as if you were really there?" on a scale ranging from 0 to 100 (Bouchard et al., 2008).

Means and standard deviation of anxiety and presence ratings are summarized in supplementary materials (Supplementary Table S1).

\section{Psychometric Questionnaires}

The German version of the State-Trait-Anxiety Inventory (STAI; Laux et al., 1981) is a self-report questionnaire assessing state anxiety with 20 items (e.g., "I am nervous.") rated on a scale from 1 ("not at all") to 4 ("very much") and assessing trait anxiety with 20 items (e.g., "I am happy.") rated on a scale from 1 ("almost never") to 4 ("almost always"). Sum scores range from 20 to 80.

The Acrophobia Questionnaire (AQ; Cohen, 1977) is a 40-item self-report questionnaire measuring fear of heights (acrophobia) on two subscales. The Anxiety subscale assesses fear towards common height situations, e.g., take a ride on the Ferris wheel, with seven-point Likert scales from 0 ("not anxious at all") to 7 ("extremely anxious"), resulting in a sum score range of $0-120$. The Avoidance subscale asks to rate the same items on a three-point Likert scale from 0 ("would not avoid") to 3 ("would not do it under any circumstances"), thus assessing behavioral avoidance in response to height situations with a sum score ranging from 0 to 40. Hüweler et al. (2009) and Diemer et al. (2016) already applied the AQ to test the acrophobic symptoms in virtual environments.

The German version of the Sensation-Seeking Scale (Beauducel et al., 2003) assesses the individual's optimal levels of stimulation and arousal with 40 items with a forced-choice design. Participants are asked to choose one of two statements that best reflect their personal beliefs (e.g., "I get bored seeing the same old faces." vs. "I like the comfortable familiarity of everyday friends."). The SSS-V has four subscales measuring "Thrill and Adventure Seeking," "Disinhibition," "Experience Seeking," and "Boredom Susceptibility." The total score ranging from 0 to 40 represents the sum score of all subscales. Due to technical problems, 18 participants were unable to answer the last five items of the SSS-V. To complete the data set, missing items were estimated by means of other items on each subscale and then rounded.

The Igroup Presence Questionnaire (IPQ; Schubert et al., 2001) consists of 14 items and evaluates the feeling of presence within the VR scenario as a self-report. Spatial Presence (e.g., "Somehow I felt, that the virtual world surrounded me."), Involvement (e.g., "I was not aware of my real environment."), and Experienced Realism (e.g., "How real did the virtual world seem to you?") are measured on a seven-point Likert Scale. Additionally, a general item assesses the "sense of being there." All subscale scores range from 0 to 6 . The IPQ has been validated for various types of virtual environments (see http://www.igroup.org/pq/ipq/index.php for further details).

Questionnaire scores (means and standard deviations) are displayed in the supplementary materials section (Supplementary Table S1).

\section{Procedure}

After signing the written informed consent, participants reported sociodemographic data and then completed the questionnaires (see above, except IPQ). Then, they entered the CAVE and completed a virtual tutorial to adapt to navigation and audio instructions.

The actual experiment started by teleporting participants to the center area of the EPM with the instruction to explore the environment for a total of $5 \mathrm{~min}$ freely. Starting gaze in the direction of one of the arms was randomized across participants. After 5 min, anxiety and presence were assessed on an open arm and a closed arm as described above. Finally, participants completed a questionnaire evaluating the sensation of presence throughout the VR exposure (IPQ).

\section{Statistical Analyses}

Statistical analyses were performed with R and SPSS (R Core Team, 2013; IBM Corp., 2016). Exploration behavior was first analyzed regarding differences between open vs. closed arms with dependent samples $t$-tests. In addition, distance walked on the various maze areas was analyzed via dependent samples $t$-test. Second, associations between exploration behavior and psychometric 


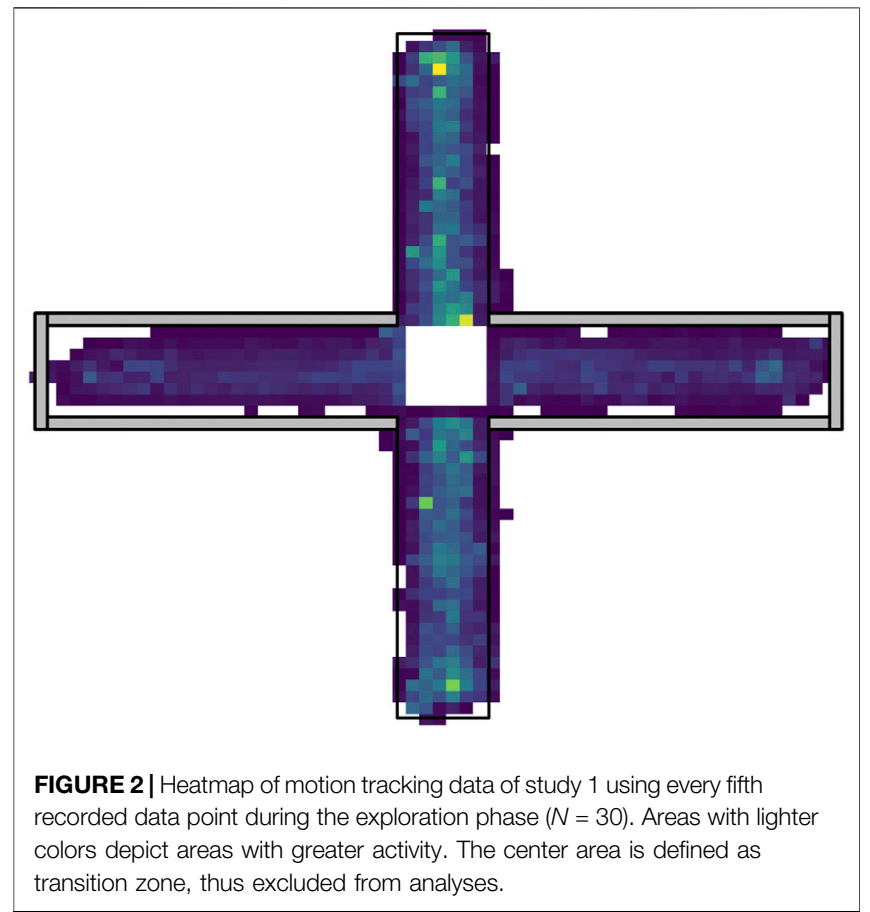

TABLE 1 | Results of correlation analyses of time spent on open or closed arms and psychometrics.

\begin{tabular}{|c|c|c|c|c|}
\hline & \multicolumn{2}{|c|}{$\begin{array}{c}\text { Time spent on open } \\
\text { arms }\end{array}$} & \multicolumn{2}{|c|}{$\begin{array}{c}\text { Time spent on closed } \\
\text { arms }\end{array}$} \\
\hline & $r$ & $p$ & $r$ & $p$ \\
\hline \multicolumn{5}{|l|}{ STAI } \\
\hline Trait & -0.38 & 0.043 & 0.17 & 0.371 \\
\hline \multicolumn{5}{|l|}{$\mathbf{A Q}$} \\
\hline Anxiety & $-0.51^{*}$ & 0.005 & 0.27 & 0.163 \\
\hline Avoidance & -0.42 & 0.020 & 0.38 & 0.037 \\
\hline \multicolumn{5}{|l|}{ SSS-V } \\
\hline Total & 0.36 & 0.048 & $-0.54^{\star}$ & 0.002 \\
\hline
\end{tabular}

Note: *Bonferroni-corrected to $p<0.0063 ; A Q$, Acrophobia Questionnaire; STAI, StateTrait-Anxiety Inventory; SSS-V, Sensation Seeking Scale.

questionnaires were analyzed with correlations; due to multiple testing, Bonferroni corrections were applied. Third, hierarchical regression analyses were performed to further evaluate the influences on open arm activity by those psychometric measures found to correlate with open arms avoidance. Finally, the difference in anxiety ratings between arms was analyzed with a $t$-test, and correlations between anxiety ratings on open and closed arms with psychometric measures were performed.

\section{Results}

\section{Exploration Behavior}

Analyses of the motion tracking data revealed that participants spent overall more time on the open $(M=151.52, S D=58.96)$ compared to the closed $(M=60.54, S D=33.39)$ arms, $t_{(29)}=$ 6.36, $p<0.001, d=1.14$ (see Figure 2). On average, subjects walked a total of $191.72 \mathrm{~m}(S D=44.85)$ throughout the
TABLE 2 | Summary of hierarchical regression analyses for psychometric questionnaire scores predicting time spent on open arms of the virtual EPM in study 1 .

\begin{tabular}{|c|c|c|c|c|c|c|}
\hline & $R^{2}$ & $A I C$ & $B$ & SE B & $\beta$ & $p$ \\
\hline Step 1 & 0.13 & 319.96 & & & & $0.033^{*}$ \\
\hline Intercept & & & 264.64 & 53.03 & & $<0.001^{\star \star}$ \\
\hline STAI Trait & & & -3.43 & 1.53 & -0.40 & $0.033^{*}$ \\
\hline Step 2 & 0.28 & 303.40 & & & & $0.006^{*}$ \\
\hline Intercept & & & 258.70 & 48.24 & & $<0.001^{\star *}$ \\
\hline STAI Trait & & & -2.37 & 1.45 & -0.28 & 0.114 \\
\hline AQ Anxiety & & & -1.61 & 0.63 & -0.43 & $0.017^{\star}$ \\
\hline Step 3 & 0.26 & 305.29 & & & & $0.018^{\star}$ \\
\hline Intercept & & & 250.91 & 75.32 & & $0.003^{\star}$ \\
\hline STAI Trait & & & -2.36 & 1.48 & -0.28 & 0.158 \\
\hline AQ Anxiety & & & -1.54 & 0.80 & -0.42 & 0.066 \\
\hline SSS-V Total & & & 0.28 & 6.38 & 0.03 & 0.892 \\
\hline
\end{tabular}

Note: ${ }^{*} p<0.05,{ }^{* *} p<0.01 ; R^{2}=$ adjusted $R^{2} ; A Q$, Acrophobia Questionnaire; STAl, State-Trait-Anxiety Inventory; SSS-V, Sensation Seeking Scale.

exploration phase and $51.89(S D=27.57)$ and $99.05(S D=$ 44.77) meters on the closed and open arms, respectively. The difference of walking distance between the two arms was significant, $t_{(29)}=-4.52, p<0.001, d=-0.83$, indicating that subjects were more active on the open than on the closed arms.

Correlational analyses (see Table 1) revealed that both trait anxiety and acrophobia (both subscales) were negatively associated with the time spent on the open arms. In contrast, the SSS-V Total score was positively correlated with time spent on open arms. However, after applying Bonferroni correction due to multiple testing $(p=0.0063)$, only the negative correlation between AQ Anxiety and time on open arms $\left(r_{(28)}=-0.51\right.$, $\mathrm{p}=0.005)$ and the negative correlation between the SSS-V and time spent on closed arms $\left(r_{(28)}=-0.54, p=0.002\right)$ remained significant, suggesting that in humans, acrophobic fear is associated with open arm avoidance, whereas proneness to sensation seeking is linked to closed arm avoidance. Correlation analyses of walking distances and psychometrics revealed no effect (all $p$ values $>0.05$ ).

A subsequent three-stage hierarchical multiple regression for time spent on open arms as the dependent variable and trait measures as predictors was conducted to evaluate the EPM behavior further. Predictors were selected based on the results of correlational analyses (see above), variables were added based on our hypotheses and correlations with the dependent variable. The test for multicollinearity indicated very low levels of multicollinearity, and other assumptions for implementation of regression models were not violated. In the first step, trait anxiety was included to test for our main hypothesis. In a second step, the Anxiety subscale of the AQ was added; the AQ Avoidance subscale was deliberately excluded due to the strong correlation between both measures $\left(r_{(27)}=0.86, p<0.001\right)$ suggesting multicollinearity. In the third step, the total score of the SSS-V was included.

Comparison of all models via adjusted $R^{2}$ score (see Table 2) indicated the second model to be best fit to explain time spent on open arms, $R_{a d j}^{2}=0.28 ; F_{2,27}=6.307, p=0.006$. In this model, fear of heights (AQ Anxiety) but not trait anxiety (STAI) significantly 
TABLE 3 | Results of correlation analyses of anxiety ratings with psychometric questionnaires.

\begin{tabular}{|c|c|c|c|c|}
\hline & \multicolumn{2}{|c|}{$\begin{array}{c}\text { Anxiety ratings on } \\
\text { open arms }\end{array}$} & \multicolumn{2}{|c|}{$\begin{array}{l}\text { Anxiety ratings on } \\
\text { closed arms }\end{array}$} \\
\hline & $r$ & $p$ & $r$ & $p$ \\
\hline \multicolumn{5}{|l|}{ STAI } \\
\hline Trait & 0.16 & 0.405 & 0.42 & 0.023 \\
\hline \multicolumn{5}{|l|}{$\mathbf{A Q}$} \\
\hline Anxiety & $0.53^{\star}$ & 0.003 & 0.32 & 0.095 \\
\hline Avoidance & $0.62^{\star}$ & $<0.001$ & 0.27 & 0.156 \\
\hline \multicolumn{5}{|l|}{ SSS-V } \\
\hline Total & -0.45 & 0.013 & -0.20 & 0.298 \\
\hline
\end{tabular}

Note: Bonferroni-corrected to $p<0.0063 ; A Q$, Acrophobia Questionnaire; STAl, StateTrait-Anxiety Inventory; SSS-V, Sensation Seeking Scale.

explained exploration behavior suggesting that, in humans, avoidance of open arms is primarily motivated by fear of heights.

\section{Anxiety Ratings}

$T$-test for dependent samples revealed significantly higher anxiety rating on open $(M=17.30, S D=20.38)$ vs. closed arms $(M=4.77, S D=6.06), t_{(29)}=3.717, p<0.001$. Further analyses of correlations between psychometrically assessed traits and anxiety ratings on open and closed arms revealed a significant positive correlation ( $p$-value was Bonferronicorrected to 0.0063 ) between anxiety rating on open arms with the AQ Anxiety $\left(r_{(28)}=0.53, \mathrm{p}=0.003\right)$ and avoidance subscales $\left(r_{(28)}=0.62, p<0.001\right)$ (see Table 3$)$. Thus, anxiety ratings on open arms increased in line with acrophobic anxiety and avoidance, whereas trait anxiety, including sensation seeking, did not seem to be notably linked to anxiety ratings on the maze.

\section{Correlation of Trait Anxiety Measures}

STAI-Trait and AQ subscale scores were uncorrelated (Anxiety: $r_{(26)}=0.29, p=0.139$; Avoidance: $\left.r_{(27)}=0.21, \mathrm{p}=0.268\right)$, while both AQ subscales were significantly negatively correlated with the SSS-V score (all $p s<0.05$ ). Overview on questionnaire intercorrelations is provided in supplementary materials (Supplementary Table S2).

\section{Summary}

Contrary to our hypothesis, we found a general open arm preference in this study. In line with our hypothesis, we found that open arm avoidance is associated with trait anxiety. However, our results revealed that avoidance of open arms was best predicted by acrophobic fear. Additionally, correlations between fear ratings on open arms and acrophobic fear and acrophobic avoidance suggest that participants with an increased trait of acrophobia experienced more fear on the open arms and therefore exhibited increased avoidance of them. The observed negative correlation between acrophobic fear and sensation seeking may explain why we also found some evidence that sensation seeking is associated with decreased time spent on closed arms and less fear on open arms, although not significant. These findings replicate the associations found by Biedermann et al. (2017), who reported similar relationships between open arm avoidance and acrophobic fear including sensation seeking, which is remarkable as the two virtual EPMs differed considerably in design. These concordant results further validate that human behavior in a virtual EPM measures anxiety, most likely acrophobic fear.

\section{STUDY 2}

Based on the findings in study 1 , we speculate that an association between EPM behavior and trait anxiety might exist but was overshadowed by a rather strong effect of acrophobic fear. Also, we found no general open arm avoidance as observed in animal studies. We think that this is due to the direct translation of the rodent EPM with closed arms with rather high walls. This restricted the view considerably and perhaps induced some claustrophobic fear, as reported by some participants postexperimentally, which might have reversed the effect and led to closed arm avoidance. Therefore, we modified the virtual EPM into a more human-adapted version and re-tested our hypotheses.

\section{Methods \\ Sample}

The final sample of 61 participants (20 males, 41 females, mostly students) had a mean age of $M=23.21$ years $(S D=3.74)$. Seven examined participants were excluded due to technical problems or premature completion of the experimental procedure. Recruitment was performed via an online platform, flyers, and emails. Participation was either monetary rewarded (12€) or compensated with course credit.

Participants were selected via online screening. For this, we extracted six items of the AQ and the Claustrophobia Questionnaire (CLQ; Radomsky et al., 2001), respectively. To assess subjects' level of acrophobia, they were asked to rate their level of fear when confronted with six typical height situations (corresponds with AQ Anxiety) on a five-point Likert scale and the extent of avoidance behavior towards them (corresponds with AQ avoidance) on a three-point Likert scale. For claustrophobic fear, the six selected items were rated on a five-point Likert scale. On a scale of sum scores ranging from 6 to 24 for acrophobic fear (but not avoidance) including claustrophobia, only participants scoring 12 or less, respectively, were invited. To examine agoraphobic fear, we asked whether the subject ever experienced a panic attack. If yes, we wanted to know whether one or more of the listed situations, e.g., wide-open places, pulled from the diagnostical criteria of the ICD-10 (Dilling and Freyberger, 2012), are avoided out of fear of experiencing another panic attack. Participants were excluded if agoraphobic tendencies were present.

Additionally, estimation of the STAI Trait score was conducted via four items with a four-point Likert scale but not considered for inclusion. 


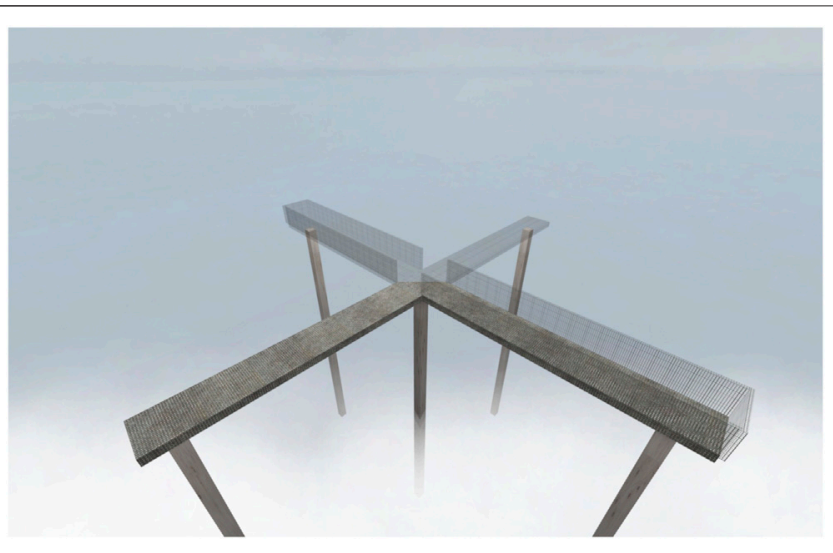

FIGURE 3 | Screenshot of the virtual EPM used in the second study. Walls on closed arms were lowered to handrail level and floor texture was modified to consist of see-through mesh and solid metal floor.

The study was carried out per the recommendations of the Declaration of Helsinki and the Ethical Guidelines of the German Psychological Society. The protocol was approved by the Ethics Committee of the Institute of Human-Computer-Interaction. All participants gave written informed consent.

\section{Apparatus and Virtual Scenario}

The apparatus was similar to that of study 1 . The virtual EPM scenario was modified as follows. Closed arms came with handrails instead of walls with a height of $1.3 \mathrm{~m}$, referring to the official German standard for the height of handrails. The arm's width and length were reduced to 1.5 and $10 \mathrm{~m}$, respectively. Finally, we changed the arms floor with one open and one closed arm having solid metal floors (as in study 1) and one open and one closed arm having see-through metal grid floors; the center area of the maze was divided diagonally and had both surface textures (see Figure 3). Please note that we were unaware of Biedermann et al.'s (2017) design as we conducted the study prior to their publication (Madeira et al., 2017).

\section{Tracking Data}

Similar to study 1, positioning data within the virtual scenario were tracked continuously with a sample rate of $60 \mathrm{~Hz}$. For this study, we calculated spent time and walked distance on each arm individually to take the various arm types (open/closed) and floor textures (solid/grid) into account. Again, we calculated the total walked distance. The center area was defined as a transition zone and therefore excluded from analyses (Hogg, 1996).

\section{Procedure and Questionnaires}

The experimental procedure was mostly similar to that of study 1 , with two exceptions. First, the Claustrophobia Questionnaire was added and completed before the experiment started. Second, anxiety ratings after the exploration trial were registered on all four arms in a randomized sequence.

The German version of the Claustrophobia Questionnaire (CLQ; Radomsky et al., 2001) evaluates fear of confined spaces with overall 26 items assessing fear of suffocation (SS; 14 items, e.g., "swimming with a nose clip") and fear of restriction (RS; 12 items, e.g., "wear handcuffs for 15 min") with five-point Likert scales from 0 ("not anxious at all") to 4 ("extremely anxious"). Sum score of the SS scale ranges from 0 to 56 , whereas sum score of the RS vary between 0 and 48 . The total questionnaire score consists of the sum score of the two subscales and ranges from 0 to 104 .

A summary of questionnaire scores, exploration, and rating data are displayed in supplementary materials (see Supplementary Table S3).

\section{Statistical Analyses}

First, differences between time spent and walking distances on EPM arms were analyzed with a $2 \times 2$ repeated measure ANOVA with the within-subject factor arm type (open vs. closed) and floor texture (solid vs. grid). Second, associations between exploration behavior and psychometric questionnaires were analyzed with correlations; due to multiple testing, Bonferroni corrections were applied. Third, hierarchical regression analyses were performed to further evaluate influences on open arms avoidance by those psychometric measures found to correlate with open arms avoidance before. Finally, differences between anxiety ratings on arms were analyzed with a $2 \times 2$ repeated measures ANOVAs with the within-subjects factors arm type (open vs. closed) and floor texture (solid vs. grid) and correlations were calculated to analyze associations between anxiety ratings on open and closed arms with psychometric measures.

\section{Results}

\section{Exploration Behavior}

Analyses of the motion tracking data (see Figure 4B) with an ANOVA with the within-subjects factors arm type (open vs. closed) and floor texture (solid vs. grid) revealed no significant main or interaction effects (all $p$ values $>0.05$ ), hence indicating no general preference of arm type or floor texture (see Figure 4). Walking distance was averaged at $62.37(S D=28.54)$ meters for closed grid arm, $53.68(S D=22.56)$ meters for closed solid, 44.47 $(S D=24.12)$ meters for open grid, and $47.19(S D=18.50)$ meters for open solid arm, respectively. In addition, the mean of total walking distance was $M=194.43(S D=49.89)$. Statistical comparison of walking distances with an ANOVA for withinsubject factor arm type and floor texture revealed a significant main effect for arm type, $F_{1,60}=4.384, p=0.041, \eta^{2}=0.068$, and a significant interaction effect, $\mathrm{F}_{1,60}=5.621, p=0.021, \eta^{2}=0.086$. No significant effect for floor type was found, $F_{1,60}=0.759$, $p=0.387, \eta^{2}=0.012$. Bonferroni-corrected post hoc $t$-tests for paired samples $(p=0.008)$ revealed a significant difference between walking distance on closed and open arm with solid floor, $t_{(60)}=-3.33, p=0.002$.

In sum, no general arm preference was observed, and walking distances differed between open and closed arms with solid floor but not between open and closed arms with grid floor or floor textures in general.

Correlational analyses (see Table 4) revealed that increase in AQ Anxiety and AQ Avoidance was associated with less time on the open arm with grid floor (Anxiety: $r_{(59)}=-0.36, p=0.004$; 
A

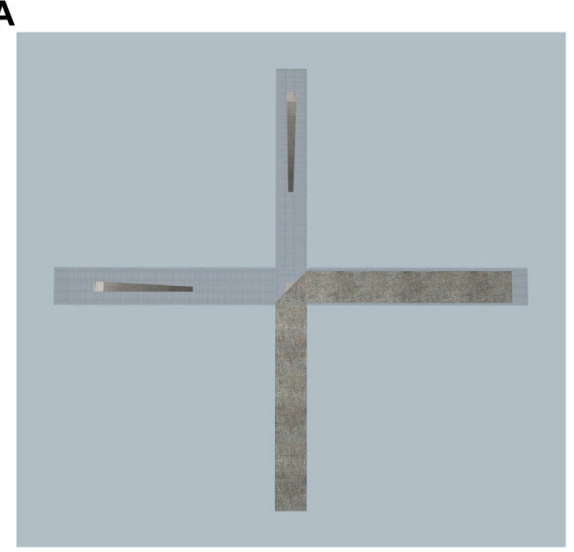

B

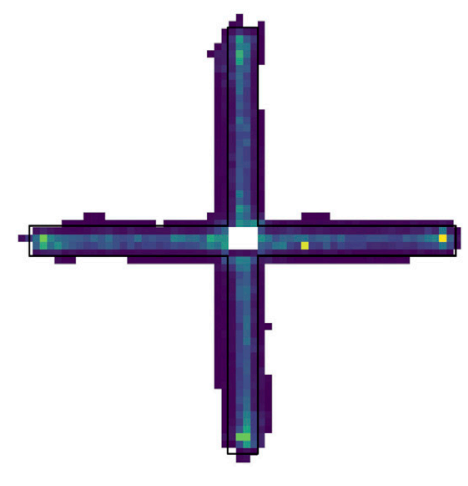

FIGURE 4 | (A) Top view screenshot of the virtual EPM depicting the different floor textures; vertical arms are open, horizontal arms are closed. (B) Heatmap of motion tracking data during the exploration phase $(N=61)$. Areas with lighter colors depict areas with greater activity.

TABLE 4 | Results of correlation analyses of time spent on various maze arms and psychometric questionnaires.

\begin{tabular}{|c|c|c|c|c|c|c|c|c|}
\hline & \multicolumn{2}{|c|}{ Closed grid } & \multicolumn{2}{|c|}{ Closed solid } & \multicolumn{2}{|c|}{ Open grid } & \multicolumn{2}{|c|}{ Open solid } \\
\hline & $r$ & $p$ & $r$ & $p$ & $r$ & $p$ & $r$ & $p$ \\
\hline \multicolumn{9}{|l|}{ STAI } \\
\hline Trait & -0.25 & 0.050 & -0.10 & 0.463 & 0.29 & 0.021 & 0.08 & 0.548 \\
\hline \multicolumn{9}{|l|}{$A Q$} \\
\hline Anxiety & 0.02 & 0.863 & 0.15 & 0.253 & -0.36 & 0.004 & -0.12 & 0.377 \\
\hline Avoidance & 0.04 & 0.768 & -0.02 & 0.859 & $-0.40^{*}$ & 0.001 & 0.02 & 0.877 \\
\hline \multicolumn{9}{|l|}{ SSS-V } \\
\hline Total & -0.19 & 0.150 & -0.09 & 0.492 & -0.13 & 0.328 & 0.06 & 0.656 \\
\hline \multicolumn{9}{|l|}{ CLQ } \\
\hline Total & 0.04 & 0.773 & -0.05 & 0.717 & -0.08 & 0.539 & -0.06 & 0.633 \\
\hline
\end{tabular}

Note: *Bonferroni-corrected to $p<0.0025 ; A Q$, Acrophobia Questionnaire; STAl, State-Trait-Anxiety Inventory; SSS-V, Sensation Seeking Scale; CLQ, Claustrophobia Questionnaire.

Avoidance: $\left.r_{(59)}=-0.40, r=0.001\right)$. Somewhat unexpected are the observations that trait anxiety was associated with more time on the open arm with grid floor $\left(r_{(59)}=0.30, p=0.021\right)$ and less time on the closed arm with grid floor $\left(r_{(59)}=-0.25, p=0.05\right)$. No effect for claustrophobic fear was detected. However, after Bonferroni correction ( $p=0.0025$ ), only the negative correlation between AQ Avoidance and time spent on the open grid floor arm remained significant $(p=0.0013)$. These findings corroborate study 1 and emphasize the importance of height perception for human EPM behavior, whereas trait anxiety and claustrophobia either play a subordinate or no role.

Moreover, a negative correlation (Bonferroni-corrected to $p=$ 0.003 ) was found between walking distance on open arm with grid floor texture and AQ Avoidance, $r_{(59)}=-0.346, p=0.001$.

A subsequent four-step hierarchical multiple regression analysis was conducted to determine predictors for time spent on open arms irrespective of floor texture, similar to study 1. Only low levels of multicollinearity were present, and there was no violation of relevant assumptions for regression analyses. The predictors selected based on the correlational analyses and study 1 findings were consecutively included in the model, i.e., trait anxiety, fear of height (AQ Anxiety), and sensation seeking (SSS-V Total). To estimate the influence of claustrophobia, we added the CLQ total score in the fourth step.

The best-fitting model according to adjusted $R^{2}$ score for predicting time spent on open arms included a linear combination of STAI-Trait and AQ-Anxiety, $R_{\text {adj }}^{2}=0.16$, $F_{2,58}=5.35, p=0.007$ (Step 2). Within this model, AQ Anxiety was the most important variable predicting the avoidance of open arms (see Table 5). These results replicate the findings of study 1 and also point to some influence of trait anxiety.

\section{Anxiety Ratings}

A $2 \times 2$ ANOVA on anxiety ratings with the within-subject factor arm type (open vs. closed), and floor texture (grid vs. solid) returned significant main effects of arm type, $F_{1,56}=27.61, p<$ $0.001, \eta^{2}=0.33$, and floor texture, $F_{1,56}=12.33, p=0.001, \eta^{2}=$ 0.18 , but no interaction effect, $F_{1,56}=0.33, p=0.569, \eta^{2}=0.01$. These findings implicate that participants rated open vs. closed arms and grid floor vs. solid floor arms as more anxiety inducing.

Further correlation analyses revealed that the anxiety reported on all EPM arms-except the "safest" with closed arm and solid floor-were positively correlated (Bonferroni-corrected $p=0.0025$ ) 
TABLE 5 | Summary of hierarchical regression analyses for psychometric questionnaire scores predicting time spent on open arms of the virtual maze in study 2.

\begin{tabular}{|c|c|c|c|c|c|c|}
\hline & $R^{2}$ & $A I C$ & $B$ & SE B & $\beta$ & $p$ \\
\hline Step 1 & 0.04 & 616.50 & & & & 0.122 \\
\hline Intercept & & & 96.62 & 18.99 & & $<0.001^{\star \star}$ \\
\hline STAI Trait & & & 0.77 & 0.49 & 0.20 & 0.122 \\
\hline Step 2 & 0.16 & 610.67 & & & & $0.007^{\star \star}$ \\
\hline Intercept & & & 111.83 & 18.76 & & $<0.001^{\star *}$ \\
\hline STAI Trait & & & 0.83 & 0.46 & 0.22 & 0.079 \\
\hline AQ Anxiety & & & -1.03 & 0.36 & -0.34 & $0.007^{\star \star}$ \\
\hline Step 3 & 0.11 & 612.56 & & & & \\
\hline Intercept & & & 118.74 & 28.87 & & $<0.001^{\star *}$ \\
\hline STAI Trait & & & 0.80 & 0.47 & 0.21 & 0.095 \\
\hline AQ Anxiety & & & -1.05 & 0.37 & -0.35 & $0.007^{\star}$ \\
\hline SSS-V Total & & & -0.27 & 0.84 & -0.04 & 0.753 \\
\hline Step 4 & 0.10 & 614.52 & & & & \\
\hline Intercept & & & 116.93 & 30.90 & & $<0.001^{\star \star}$ \\
\hline STAI Trait & & & 0.80 & 0.48 & 0.21 & 0.098 \\
\hline AQ Anxiety & & & -1.07 & 0.40 & -0.36 & $0.010^{\star}$ \\
\hline SSS-V Total & & & -0.23 & 0.87 & -0.04 & 0.791 \\
\hline CLQ Total score & & & 0.08 & 0.43 & 0.02 & 0.862 \\
\hline
\end{tabular}

Note: ${ }^{*} p<0.05,{ }^{* *} p<0.01 ; R^{2}=$ adjusted $R^{2} ; A Q$, Acrophobia Questionnaire; STAl, State-Trait-Anxiety Inventory; SSS-V, Sensation Seeking Scale.

with both AQ subscales (see Table 6). Furthermore, a significant negative relationship was found between SSS-V Total score and all arms except the open arm with a grid floor. Finally, significant positive correlations were found between the CLQ total score and anxiety ratings on all arms, whereas correlations between STAI-Trait and anxiety ratings did not reach significance.

\section{Correlations of Trait Anxiety Measures}

Replicating study 1 , we found again that AQ scores of both subscales were uncorrelated with STAI-Trait (Anxiety: $r_{(59)}=0.04, p=0.715$, Avoidance: $\left.r_{(59)}=0.12, p=0.347\right)$. Furthermore, we observed that both AQ Anxiety and AQ Avoidance were positively associated with the CLQ total score (Anxiety: $r_{(59)}=0.39, p=0.002$; Avoidance: $\left.r_{(59)}=0.43, p=0.001\right)$. In addition, CLQ total score and SSS-V-Total score correlated negatively, $r_{(59)}=-0.28, p=0.028$ (see Supplementary Table S4 in supplementary materials). This suggests that in this sample acrophobia is associated with claustrophobia whereas sensation seeking is not or even reversely linked to phobic anxiety.

\section{Summary}

With respect to the first hypothesis for this study, we again found no general open arm avoidance in humans and no area preference in general. Finally, the findings on fear ratings assessed on the various EPM arms replicated study 1 . Acrophobia but not trait anxiety positively related to fear on all maze arms, except the least "acrophobic" one, i.e., the closed arm with a solid floor. Also, sensation seeking was in general negatively associated with fear ratings on EPM arms although only the associations with ratings on the closed arm with grid floor and open arm with solid floor survived significance. Unexpectedly, we observed that claustrophobia was positively associated with fear ratings on all areas of the maze, which may be due to the claustrophobic situation of being in the CAVE system.

In sum, these results confirm the importance of acrophobia as the driving factor for human EPM behavior, while trait anxiety seems to play a rather subordinate role in open arm activity if any.

\section{DISCUSSION}

In the two studies, we conceptually transferred the EPM, a wellestablished animal model of anxiety measurement, to a human context using virtual reality technology. Equally to animal studies (Pellow et al., 1985), the participants' behaviors during $5 \mathrm{~min}$ of free exploration of a virtual copy of an animal EPM adjusted to human proportions (study 1) or a human-adapted EPM (study 2) were registered. The construct validity of human EPM behavior as a measure of anxiety was evaluated by testing associations with psychometric measures of anxiety or sensation seeking and fear ratings.

\section{Exploration Behavior}

Both of our studies observed no general open arm avoidance of humans, which we expected based on animal studies and the human EPM study of Biedermann et al. (2017). As a result, this finding implies that exploration behavior on the animal and human EPM is driven by mechanisms different from those in the animal EPM. Unlike the human open field test, which reproduced human thigmotaxis, we could not produce similar evolutionary conserved behaviors (Walz et al., 2016). Therefore, we conclude that we have no convincing evidence for crossspecies open arm avoidance suggested by Biedermann et al. (2017).

TABLE 6 | Results of correlation analyses of anxiety ratings on various EPM arms with psychometric questionnaires in study 2.

\begin{tabular}{|c|c|c|c|c|c|c|c|c|}
\hline & \multicolumn{2}{|c|}{ Closed grid } & \multicolumn{2}{|c|}{ Closed solid } & \multicolumn{2}{|c|}{ Open grid } & \multicolumn{2}{|c|}{ Open solid } \\
\hline & $r$ & $p$ & $r$ & $p$ & $r$ & $p$ & $r$ & $p$ \\
\hline \multicolumn{9}{|l|}{ STAI } \\
\hline Trait & 0.05 & 0.712 & 0.18 & 0.171 & 0.01 & 0.921 & 0.13 & 0.309 \\
\hline \multicolumn{9}{|l|}{$\mathbf{A Q}$} \\
\hline Anxiety & $0.57^{\star}$ & $<0.001$ & 0.36 & 0.004 & $0.49^{\star}$ & $<0.001$ & $0.49^{\star}$ & $<0.001$ \\
\hline Avoidance & $0.46^{\star}$ & $<0.001$ & 0.34 & 0.008 & $0.51^{*}$ & $<0.001$ & $0.50^{\star}$ & $<0.001$ \\
\hline \multicolumn{9}{|l|}{ SSS-V } \\
\hline Total & $-0.43^{\star}$ & $<0.001$ & $-0.38^{*}$ & 0.002 & -0.38 & 0.003 & $-0.45^{\star}$ & $<0.001$ \\
\hline \multicolumn{9}{|l|}{ CLQ } \\
\hline Total & $0.45^{\star}$ & $<0.001$ & $0.42^{\star}$ & $<0.001$ & $0.43^{*}$ & $<0.001$ & $0.45^{\star}$ & $<0.001$ \\
\hline
\end{tabular}

Note: * ${ }^{p}$ < 0.0025. STAl, State-Trait-Anxiety Inventory; AQ, Acrophobia Questionnaire; SSS-V, Sensation Seeking Scale; CLQ, Claustrophobia Questionnaire. 
Several differences between ours and the study of Biedermann et al. (2017) might explain the discrepancy. At first, the definition of the EPM zones differed from each other. The exploration activity in the center area center activity remains a highly discussed topic in animal research. It was found that it merely associates with general locomotor activity but not anxiety (Braun et al., 2011). Thus we decided not to incorporate center movement into our final analyses. In addition, we followed the advice of other authors to not merge maze areas for data analysis (Hogg, 1996; Carobrez and Bertoglio, 2005; Braun et al., 2011). Nonetheless, Biedermann et al. (2017) defined their maze's closed and center area as a "safe area". Consequently, comparing a rather large (safe) area to a much smaller area (open arms) likely leads to an overestimation of time spent on these safe areas. In addition, increased closed arm activity might also be the result of perceived dangerousness in the context of the height situation. Considering that human EPM behavior is mainly modulated by acrophobic fear, it seems conceivable that the mixed virtual reality design using a wooden cross with an arm width of $30 \mathrm{~cm}$ and a height of $20 \mathrm{~cm}$ amplifies fear in reaction to height cues, which prompted a stronger avoidance of open arms than we observed in our studies.

\section{Exploration Behavior and Anxiety}

Both of our human EPM studies identified acrophobia to be the dominant factor modulating open arm avoidance. Our results corroborate Biedermann et al.'s (2017), who also reported modulatory effects of acrophobia on open arm avoidance and also added further insight. First, the modulatory effects of acrophobia on human EPM behavior are observable for elevated mazes of quite various designs, but visual height perception seems to be a crucial factor in humans. Thus, the strongest associations between acrophobia and open arm avoidance were observed in EPMs with strong height simulation, i.e., in our study 2 with an open arm with a see-through mesh ground and in Biedermann et al. (2017) comparing elevated arms of $55 \mathrm{~m}$ height with safe arms. A priori screening excluded participants with acrophobic fear with the goal to unravel trait anxiety effects on EPM behavior. Nonetheless, acrophobic fear was still the strongest modulator of human EPM behavior as revealed by correlational and hierarchical regression analyses. Second, our studies including Biedermann et al. (2017) demonstrated that sensation seeking also has a modulatory role for human EPM behavior. We observed that an increase in sensation seeking is associated with less time on closed arms. In contrast, Biedermann et al. (2017) reported that subjects with higher sensation seeking levels tend to spend more time on open arms. However, it remains questionable whether these effects can be seen independent of acrophobia as both questionnaires correlate negatively. Supporting this view, we noticed that several items of the SSS-V (Zuckerman, Eysenck, and Eysenck, 1978) refer to typical acrophobic situations (e.g., "I like to dive off the high board." vs "I don't like the feeling I get standing on the high board (or I don't go near it at all)."). Further studies have to evaluate whether acrophobia and sensation seeking modulate human EPM behavior independently. Third, closed arms with walls may be a safe area for rodents, but not for humans, according to our studies. We speculate that humans feel confined in such hallways and therefore are motivated to leave them. The replacement of high solid walls by natural handrails allowing the overview of the virtual environment may have reduced the participants' motivation (e.g., due to claustrophobia or curiosity) to leave the closed arms. Finally, we found some evidence for a modulatory effect of trait anxiety on human EPM behavior. However, these effects were weak and not consistent across the two studies. This is in line with that of Biedermann et al. (2017) who also found no modulatory role of trait anxiety. Newer studies suggest that transdiagnostic concepts like fear of the unknown (FOTU) and intolerance of uncertainty (IU) are underlying mechanisms of trait anxiety, which are easier to target in an experimental setting (Carleton et al., 2012; Carleton, 2016; Shihata et al., 2016). Therefore, we suggest that future studies introduce instruments to examine FOTU or IU (Carleton et al., 2007) to gain further insights into nonphobic anxiety mechanisms in a human EPM. To sum it up, we have to conclude that, based on the existing evidence, the human virtual EPM is instead a behavioral test for acrophobia than for anxiety in general.

\section{Anxiety Ratings}

The anxiety ratings assessed on different EPM arms partly mirrored the association of acrophobia and behavioral movement measures. For example, participants reported enhanced anxiety on open vs. closed arms and arms with see-through mesh floor vs. solid floor, emphasizing the importance of visual height perception again. In line, these ratings were associated with acrophobia but not trait anxiety, and we found some negative associations between sensation seeking and anxiety rating. In sum, these results suggest that acrophobia and, to some extent sensation, seeking, modulate human EPM behavior via the anxiety experienced on different EPM arms. Interestingly, claustrophobic tendencies were associated with anxiety ratings on all arms. Therefore, we speculate that the five-sided CAVE system is a claustrophobic context intrinsically, as we did not observe a consistent distinction between arm types or floor texture in the second study. Consequently, future studies have to consider and evaluate how the technology used to present virtual environments moderates the experienced anxiety and perhaps human EPM behavior.

\section{Limitations}

Our studies assessed behavioral and verbal measures of anxiety but lack psychophysiological measures. Future studies should consider psychophysiological measures as they may indicate anxiety responses independent of self-report or behavioral measures (Lang, 1985). In addition, more detailed analyses of movement patterns should be considered. Besides exploration time as assessed here, rodent EPM studies also assess other fear-like responses, such as freezing. In humans, freezing is defined as reduced mobility and bradycardia and has already been observed in specific human experimental settings (Roelofs et al., 2010; Hagenaars et al., 2014; Rösler and Gamer, 2019). Assessing freezing as human EPM behavior would be especially valuable as participants may enter open arm alleys but abruptly freeze, increasing the time spent on that specific maze area leading to erroneous interpretation of results.

Besides, the generalization of these findings might be limited by the small age range within the sample consisting predominantly of students. 
Finally, several participants reported that the virtual environments appeared somewhat artificial. Thus, future studies should think about naturalistic EPM environments to increase the test's validity.

\section{CONCLUSION}

Our studies show that human movement in a virtual human EPM is primarily influenced by acrophobia but not by trait anxiety, as we initially hypothesized. The results are even more interesting, as, in the second study, participants were selected for low levels of acrophobia to avoid distortion of results. To address the influence of trait anxiety on exploration behavior, it is essential to reshape the virtual EPM in future studies to avoid the influence of fear of heights. To target trait anxiety-related processes, we suggest the subliminal induction of a threat to trigger a generalized fear state typically associated with high trait anxiety (e.g., Van Den Hout et al., 1995; Li et al., 2007; Reuman et al., 2015). This can either be achieved by a threat of shock paradigm (Clark et al., 2012; Morriss et al., 2019) or by emotional priming (Yang et al., 2016).

The present studies examined the influence of anxiety and personality traits on human exploration behavior in detail. In conclusion, we consider the virtual EPM an essential apparatus in the investigation of anxiety-related issues as the virtual scenario can easily be modified.

\section{DATA AVAILABILITY STATEMENT}

The original contributions presented in the studies can be found on https://osf.io/j2fsw/. Further inquiries can be directed to the corresponding author.

\section{ETHICS STATEMENT}

The studies involving human participants were reviewed and approved by the Ethical Board of the Department of Human-

\section{REFERENCES}

Aupperle, R. L., Sullivan, S., Melrose, A. J., Paulus, M. P., and Stein, M. B. (2011). A reverse translational approach to quantify approach-avoidance conflict in humans. Behav. Brain Res. 225 (2), 455-463. doi:10.1016/j.bbr. 2011.08.003

Barnett, S. A. (2017). The rat: a study in behavior. London, United Kingdom: Routledge.

Beauducel, A., Strobel, A., and Brocke, B. (2003). Psychometrische eigenschaften und normen einer deutschsprachigen fassung der sensation seeking-skalen, form V. Diagnostica 49 (2), 61-72. doi:10.1026/0012-1924.49.2.61

Biedermann, S. V., Biedermann, D. G., Wenzlaff, F., Kurjak, T., Nouri, S., Auer, M. K., et al. (2017). An elevated plus-maze in mixed reality for studying human anxiety-related behavior. BMC Biol. 15 (1), 125. doi:10.1186/s12915017-0463-6

Boecker, L., and Pauli, P. (2019). Affective startle modulation and psychopathology: implications for appetitive and defensive brain systems. Neurosci. Biobehavioral Rev. 103, 230-266. doi:10.1016/j.neubiorev.2019.05.019
Computer-Interaction of the University of Würzburg. All participants provided their written informed consent to participate in this study.

\section{AUTHOR CONTRIBUTIONS}

OM, DG, MEL, and PP contributed to the study concept and design. Data collection was done by OM. OM and DG performed data analysis and interpretation. OM and PP drafted the manuscript, and all other co-authors provided critical revisions and feedback. All authors contributed to the article and approved the submitted version.

\section{FUNDING}

The studies were supported by the Volkswagen Foundation (AZ 94 102). This work was carried out with the support of the structural doctoral program "Graduate School of Life Sciences (GSLS)" by the University of Würzburg, and the two studies are part of the doctoral project "The humanexperimental virtual Elevated Plus-Maze as an anxiety model" of OM. This publication was supported by the Open Access Publication Fund of the University of Wuerzburg.

\section{ACKNOWLEDGMENTS}

We would like to thank Jan Philipp Gast for assisting in data collection and preparation.

\section{SUPPLEMENTARY MATERIAL}

The Supplementary Material for this article can be found online at: https:/www.frontiersin.org/articles/10.3389/frvir.2021.635048/ full\#supplementary-material.

Bohil, C. J., Alicea, B., and Biocca, F. A. (2011). Virtual reality in neuroscience research and therapy. Nat. Rev. Neurosci. 12 (12), 752-762. doi:10.1038/ nrn3122

Bouchard, S., St-Jacques, J., Robillard, G., and Renaud, P. (2008). Anxiety increases the feeling of presence in virtual reality. Teleoperators Virtual Environ. 17 (4), 376-391. doi:10.1162/pres.17.4.376

Braun, A. A., Skelton, M. R., Vorhees, C. V., and Williams, M. T. (2011). Comparison of the elevated plus and elevated zero mazes in treated and untreated male Sprague-Dawley rats: effects of anxiolytic and anxiogenic agents. Pharmacol. Biochem. Behav. 97 (3), 406-415. doi:10.1016/j.pbb.2010.09.013

Carleton, N. R. (2016). Fear of the unknown: one fear to rule them all?. J. Anxiety Disord. 41, 5-21. doi:10.1016/j.janxdis.2016.03.011

Carleton, R. N., Peter, M. A., Norton, J., and Asmundson, G. J. (2007). Fearing the unknown: a short version of the intolerance of uncertainty scale. J. Anxiety Disord. 21 (1), 105-117. doi:10.1016/j.janxdis.2006.03.014

Carleton, R. N., Mulvogue, M. K., Thibodeau, M. A., McCabe, R. E., Antony, M. M., and Asmundson, G. J. (2012). Increasingly certain about uncertainty: intolerance of uncertainty across anxiety and depression. J. Anxiety Disord. 26 (3), 468-479. doi:10.1016/j.janxdis.2012.01.011 
Carobrez, A. P., and Bertoglio, L. J. (2005). Ethological and temporal analyses of anxiety-like behavior: the elevated plus-maze model 20 years on. Neurosci. Biobehav Rev. 29 (8), 1193-1205. doi:10.1016/j.neubiorev.2005.04.017

Carter, R. M., Wittchen, H. U., Pfister, H., and Kessler, R. C. (2001). One-year prevalence of subthreshold and threshold DSM-IV generalized anxiety disorder in a nationally representative sample. Depress. Anxiety 13 (2), 78-88. doi:10. 1002/da. 1020

Clark, L., Li, R., Wright, C. M., Rome, F., Fairchild, G., Dunn, B. D., et al. (2012). Risk-avoidant decision making increased by threat of electric shock. Psychophysiology 49 (10), 1436-1443. doi:10.1111/j.1469-8986.2012. 01454.x

Cohen, D. C. (1977). Comparison of self-report and overt-behavioral procedures for assessing acrophobia. Behav. Ther. 8 (1), 17-23. doi:10.1016/S00057894(77)80116-0

Diemer, J., Lohkamp, N., Mühlberger, A., and Zwanzger, P. (2016). Fear and physiological arousal during a virtual height challenge--effects in patients with acrophobia and healthy controls. J. Anxiety Disord. 37, 30-39. doi:10.1016/j. janxdis.2015.10.007

Dilling, H., and Freyberger, H. J. (2012). Taschenführer zur ICD-10-Klassifikation psychischer Störungen. Berlin, Heidelberg: Springer.

Dobricki, M., and Pauli, P. (2016). Sensorimotor body-environment interaction serves to regulate emotional experience and exploratory behavior. Heliyon 2 (10), e00173. doi:10.1016/j.heliyon.2016.e00173

Gallagher, M. W., Bentley, K. H., and Barlow, D. H. (2014). Perceived control and vulnerability to anxiety disorders: a meta-analytic review. Cogn. Ther. Res. 38 (6), 571-584. doi:10.1007/s10608-014-9624-x

Gould, T. D., Dao, D. T., and Kovacsics, C. E. (2009). “The open field test," in Mood and anxiety related phenotypes in mice: characterization using behavioral tests. Editor T. D. Gould (Totowa, NJ: Humana Press), 1-20.

Griebel, G., and Holmes, A. (2013). 50 years of hurdles and hope in anxiolytic drug discovery. Nat. Rev. Drug Discov. 12 (9), 667-687. doi:10.1038/nrd4075

Grillon, C., and Ernst, M. (2016). Gain in translation: is it time for thigmotaxis studies in humans?. Biol. Psychiatry 80 (5), 343-344. doi:10.1016/j.biopsych. 2016.07.003

Grillon, C., Robinson, O. J., Cornwell, B., and Ernst, M. (2019). Modeling anxiety in healthy humans: a key intermediate bridge between basic and clinical sciences. Neuropsychopharmacol. 44 (12), 1999-2010. doi:10.1038/s41386019-0445-1

Gromer, D., Reinke, M., Christner, I., and Pauli, P. (2019). Causal interactive links between presence and fear in virtual reality height exposure. Front. Psychol. 10 (141), 141. doi:10.3389/fpsyg.2019.00141

Haaker, J., Maren, S., Andreatta, M., Merz, C. J., Richter, J., Richter, S. H., et al. (2019). Making translation work: harmonizing cross-species methodology in the behavioural neuroscience of Pavlovian fear conditioning. Neurosci. Biobehavioral Rev. 107, 329-345. doi:10.1016/j.neubiorev.2019.09.020

Hagenaars, M. A., Oitzl, M., and Roelofs, K. (2014). Updating freeze: aligning animal and human research. Neurosci. Biobehav Rev. 47, 165-176. doi:10.1016/ j.neubiorev.2014.07.021

Hofmann, S. G., Alpers, G. W., and Paul, P. (2009). "Phenomenology of panic and phobic disorders," in Oxford library of psychology. Oxford handbook of anxiety and related disorders. Editors M. M. Antony and M. B. Stein (New York, NY: Oxford University Press), 34-46.

Hogg, S. (1996). A review of the validity and variability of the elevated plus-maze as an animal model of anxiety. Pharmacol. Biochem. Behav. 54 (1), 21-30. doi:10. 1016/0091-3057(95)02126-4

Hüweler, R., Kandil, F. I., Alpers, G. W., and Gerlach, A. L. (2009). The impact of visual flow stimulation on anxiety, dizziness, and body sway in individuals with and without fear of heights. Behav. Res. Ther. 47 (4), 345-352. doi:10.1016/j. brat.2009.01.011

IBM Corp. (2016). IBM SPSS statistics for windows, version 24.0. Armonk, NY: IBM Corp.

Karsten, J., Nolen, W. A., Penninx, B. W., and Hartman, C. A. (2011). Subthreshold anxiety better defined by symptom self-report than by diagnostic interview. J. Affect Disord. 129 (1), 236-243. doi:10.1016/j.jad.2010.09.006

Kinateder, M., Ronchi, E., Gromer, D., Müller, M., Jost, M., Nehfischer, M., et al. (2014). Social influence on route choice in a virtual reality tunnel fire. Transp. Res. F Traffic Psychol. Behav. 26, 116-125. doi:10.1016/j.trf.2014.06.003
Kindt, M., and Soeter, M. (2014). Fear inhibition in high trait anxiety. PLoS One 9 (1), e86462. doi:10.1371/journal.pone.0086462

Kirlic, N., Young, J., and Aupperle, R. L. (2017). Animal to human translational paradigms relevant for approach avoidance conflict decision making. Behav. Res. Ther. 96, 14-29. doi:10.1016/j.brat.2017.04.010

Komada, M., Takao, K., and Miyakawa, T. (2008). Elevated plus maze for mice. J. Vis. Exp. 22, e1088. doi:10.3791/1088

Lang, P. J. (1985). "The cognitive psychophysiology of emotion: fear and anxiety," in Anxiety and the anxiety disorders. Editors A. Hussain Tuma and J. D. Maser (Hillsdale, NJ: Lawrence Erlbaum Associates), 131-170.

Laux, L., Glanzmann, P., Schaffner, P., and Spielberger, C. D. (1981). State-trait anxiety inventory - manual of the German version. Weinheim, Germany: Beltz.

Li, W., Zinbarg, R. E., and Paller, K. A. (2007). Trait anxiety modulates supraliminal and subliminal threat: brain potential evidence for early and late processing influences. Cogn. Affect Behav. Neurosci. 7 (1), 25-36. doi:10. 3758/cabn.7.1.25

Lonsdorf, T. B., Menz, M. M., Andreatta, M., Fullana, M. A., Golkar, A., Haaker, J., et al. (2017). Don't fear "fear conditioning": methodological considerations for the design and analysis of studies on human fear acquisition, extinction, and return of fear. Neurosci. Biobehavioral Rev. 77, 247-285. doi:10.1016/j. neubiorev.2017.02.026

Madeira, O., Gromer, D., and Paul, P. (2017). "Transferring the elevated plus-maze to a human context: a virtual reality study," in Journal of neural transmission. (Wien, Austria: Springer Wien), 1309.

Maner, J. K., and Schmidt, N. B. (2006). The role of risk avoidance in anxiety. Behav. Ther. 37 (2), 181-189. doi:10.1016/j.beth.2005.11.003

Montgomery, K. C. (1955). The relation between fear induced by novel stimulation and exploratory behavior. J. Comp. Physiol. Psychol. 48 (4), 254-260. doi:10. 1037/h0043788

Morriss, J., Saldarini, F., Chapman, C., Pollard, M., and van Reekum, C. M. (2019). Out with the old and in with the new: the role of intolerance of uncertainty in reversal of threat and safety. J. Exp. Psychopathol. 10 (1), 204380871983445. doi:10.1177/2043808719834451

Pellow, S., Chopin, P., File, S. E., and Briley, M. (1985). Validation of open:closed arm entries in an elevated plus-maze as a measure of anxiety in the rat. J. Neurosci. Methods 14 (3), 149-167. doi:10.1016/0165-0270(85)90031-7

$\mathrm{R}$ Core Team (2013). $R$ : a language and environment for statistical computing. Vienna, Austria: R Core Team.

Radomsky, A. S., Rachman, S., Thordarson, D. S., McIsaac, H. K., and Teachman, B. A. (2001). The claustrophobia questionnaire. J. Anxiety Disord. 15 (4), 287-297. doi:10.1016/S0887-6185(01)00064-0

Ramos, A., Pereira, E., Martins, G. C., Wehrmeister, T. D., and Izídio, G. S. (2008). Integrating the open field, elevated plus maze and light/dark box to assess different types of emotional behaviors in one single trial. Behav. Brain Res. 193 (2), 277-288. doi:10.1016/j.bbr.2008.06.007

Rebenitsch, L., and Owen, C. (2016). Review on cybersickness in applications and visual displays. Virtual Reality 20 (2), 101-125. doi:10.1007/s10055-016-0285-9

Reuman, L., Jacoby, R. J., Fabricant, L. E., Herring, B., and Abramowitz, J. S. (2015). Uncertainty as an anxiety cue at high and low levels of threat. J. Behav. Ther. Exp. Psychiatry 47, 111-119. doi:10.1016/j.jbtep.2014.12.002

Roelofs, K., Hagenaars, M. A., and Stins, J. (2010). Facing freeze: social threat induces bodily freeze in humans. Psychol. Sci. 21 (11), 1575-1581. doi:10.1177/ 0956797610384746

Rösler, L., and Gamer, M. (2019). Freezing of gaze during action preparation under threat imminence. Sci. Rep. 9 (1), 17215. doi:10.1038/s41598-019-53683-4

Sanchez-Vives, M. V., and Slater., M. (2005). From presence to consciousness through virtual reality. Nat. Rev. Neurosci. 6 (4), 332-339. doi:10.1038/nrn1651

Schubert, T., Friedmann, F., and Regenbrecht, H. (2001). Igroup presence questionnaire. Teleoperators Virtual Environ. 41, 115-124. doi:10.1162/105474601300343603

Shihata, S., McEvoy, P. M., Mullan, B. A., and Carleton, R. (2016). Intolerance of uncertainty in emotional disorders: what uncertainties remain?. J. Anxiety Disord. 41, 115-124. doi:10.1016/j.janxdis.2016.05.001

Spielberger, C. D. (2013). Anxiety: current trends in theory and research. Amsterdam, Netherlands: Elsevier.

Spielberger, C. D. (1966). Anxiety Behavior. Cambridge, MA: Academic Press.

Treit, D., Menard, J., and Royan, C. (1993). Anxiogenic stimuli in the elevated plus-maze. Pharmacol. Biochem. Behav. 44 (2), 463-469. doi:10.1016/0091-3057(93)90492-c 
Van Den Hout, M., Tenney, N., Huygens, K., Merckelbach, H., and Kindt, M. (1995). Responding to subliminal threat cues is related to trait anxiety and emotional vulnerability: a successful replication of MacLeod and Hagan (1992). Behav. Res. Ther. 33 (4), 451-454. doi:10.1016/0005-7967(94)00062-o

Walz, N., Mühlberger, A., and Pauli, P. (2016). A human open field test reveals thigmotaxis related to agoraphobic fear. Biol. Psychiatry 80 (5), 390-397. doi:10. 1016/j.biopsych.2015.12.016

Yang, Y., Lueken, U., Wittmann, A., Holtz, K., Kleint, N. I., Herrmann, M. J., et al. (2016). Neural correlates of individual differences in anxiety sensitivity: an fMRI study using semantic priming. Soc. Cogn. Affect. Neurosci. 11 (8), 1245-1254. doi:10.1093/scan/nsw024

Zuckerman, M., Eysenck, S., and Eysenck, H. J. (1978). Sensation seeking in England and America: cross-cultural, age, and sex comparisons. J. Consult Clin. Psychol. 46 (1), 139-149. doi:10.1037//0022-006x.46.1.139
Conflict of Interest: PP is a shareholder of a commercial company that develops virtual environment research systems (VTplus $\mathrm{GmbH}$ ) for empirical studies in the field of psychology, psychiatry, and psychotherapy.

The remaining authors declare that the research was conducted in the absence of any commercial or financial relationships that could be construed as a potential conflict of interest.

Copyright (C) 2021 Madeira, Gromer, Latoschik and Pauli. This is an open-access article distributed under the terms of the Creative Commons Attribution License (CC $B Y)$. The use, distribution or reproduction in other forums is permitted, provided the original author(s) and the copyright owner(s) are credited and that the original publication in this journal is cited, in accordance with accepted academic practice. No use, distribution or reproduction is permitted which does not comply with these terms. 